\title{
Estimation of Vapor Quality at Compressor Suction of
}

\section{Air Conditioner}

\author{
Kazuhiro Endoh \\ Hitachi Research Laboratory, Hitachi, Ltd., Ibaraki 312-0034, Japan
}

Received: January 05, 2014 / Accepted: March 04, 2015 / Published: April 30, 2015.

\begin{abstract}
To evaluate performance and reliability of an air conditioner, the states of its refrigerant in the refrigeration cycle need to be understood. Since the isentropic exponent of the next-generation refrigerant R32 is larger than that of conventional refrigerant R410A, the compressor discharge temperature of R32 is higher than that of R410A. When a wet refrigerant is entered into a compressor suction line to decrease the discharge temperature, it is generally difficult to estimate the vapor quality at the suction. This paper describes a method that estimates a vapor quality at the compressor suction by applying energy balances on the compressor with the values of suction pressure, discharge pressure, discharge temperature, etc. In the air conditioner test, the vapor qualities at the compressor suction estimated with this method agreed well with those estimated with the conventional method by measuring liquid and vapor flow-rates. Moreover, this paper derived a relational expression for estimating a compressor suction state from a discharge state and discusses the characteristics of the coefficients in the expression calculated from the test results.
\end{abstract}

Key words: Air conditioner, compressor, wet refrigerant, vapor quality, R32.

\section{Introduction}

HFCs (hydrofluorocarbons), which have been used as refrigerants, have high GWPs (global warming potentials). They were thus designated as emission control substances under the Kyoto Protocol in 1997. In 2014, the EU (European Union) adopted revised F-gas regulation, which includes $\mathrm{CO}_{2}$ equivalent phase-down of HFCs to be placed on the EU market [1]. In Japan, revised fluorocarbon law was enacted in 2013 and will come into force in 2015 to promote the use of lower GWP fluorocarbons [2]. Under these circumstances, room air conditioners using the next-generation refrigerant $\mathrm{R} 32(\mathrm{GWP}=675)$, whose GWP is one third that of a conventional refrigerant R410A ( $G W P=2,088)$, have been commercialized in Japan [3]. Since the isentropic exponent of R32 is

Corresponding author: Kazuhiro Endoh, senior researcher, research fields: refrigeration and air conditioning. E-mail: kazuhiro.endo.un@hitachi.com.

This paper was presented at 15 th International Refrigeration and Air Conditioning Conference at Purdue, July 14-17, 2014, West Lafayette, IN, USA. larger than that of R410A, the compressor discharge temperature of R32 is higher than that of R410A [4]. The conventional way to decrease the discharge temperature is to enter the wet (two-phase vapor-liquid) refrigerant into the compressor suction line [5]. In the compressor test setup that connects a bypass line from the condenser outlet to the compressor suction line, a vapor quality, which represents the state of wet refrigerant, at the compressor suction can be set with flow-rates and enthalpies of vapor from the evaporator outlet and liquid from the bypass line $[6,7]$. However, it is generally difficult to estimate a vapor quality at the compressor suction during the practical operation of an air conditioner.

This paper aims at estimation of a vapor quality at the compressor suction by applying energy balances on the compressor with the values of suction pressure, discharge pressure, discharge temperature, etc. Moreover, this paper derives a relational expression for estimating a compressor suction state from a discharge state and discusses the characteristics of the 
coefficients in the expression calculated from the test results.

\section{Estimation of Vapor Quality at Compressor Suction}

\subsection{Estimation of Suction Quality by Applying Energy}

\section{Balances on Compressor}

Vapor quality $x$ is defined as the ratio of the total mass of two-phase vapor-liquid refrigerant $m$ and the mass of saturated vapor $m^{\prime \prime}$ :

$$
x=\frac{m^{\prime \prime}}{m}
$$

Quality $x$ can also be expressed as the ratio of the total mass flow-rate of two-phase vapor-liquid refrigerant $G_{r}$ and the mass flow-rate of saturated vapor $G_{r}{ }^{\prime \prime}$ :

$$
x=\frac{G_{r} "}{G_{r}}
$$

Moreover, quality $x$ is given in terms of the enthalpy of two-phase vapor-liquid refrigerant $h$, the enthalpy of saturated liquid $h^{\prime}$, and the enthalpy of saturated vapor $h^{\prime \prime}$ :

$$
x=\frac{h-h^{\prime}}{h^{\prime \prime}-h^{\prime}}
$$

Since the mass $m^{\prime \prime}$ and the mass flow-rate $G_{r}$ " of saturated vapor in Eqs. (1) and (2) cannot be measured directly, a method to estimate the quality at the compressor suction will be provided by using the relationship between a quality and enthalpies given in Eq. (3).

A suction quality $x_{s}$ is expressed by Eq. (3), using index $s$ :

$$
x_{s}=\frac{h_{s}-h_{s}{ }^{\prime}}{h_{s}{ }^{\prime}-h_{s}{ }^{\prime}}
$$

With Eq. (4), even when the suction state is superheated vapor, the state can be represented as a number $(>1.0)$.

Fig. 1 shows the $P$ - $h$ diagram for representing the relationships between enthalpies at the compressor suction and discharge. The method to estimate a suction quality $x_{s}$ by applying energy balances on the compressor is described below. This method uses the values of a suction pressure $P_{s}$, a discharge pressure $P_{d}$, a discharge temperature $T_{d}$, a refrigerant mass flow-rate $G_{r}$, a compressor power consumption $W_{c}$, and a compressor ambient temperature $T_{a}$ during the practical operation of the air conditioner. A suction quality $x_{s}$ will be identified in such a way that a discharge enthalpy $h_{d 2}$ estimated from an assumed suction quality $x_{s}$ by using energy balances is the same as the enthalpy $h_{d}$ calculated from the discharge pressure $P_{d}$ and temperature $T_{d}$.

A suction enthalpy $h_{s}$ is represented as follows from Eq. (4), assuming the value of the quality $x_{s}$ :

$$
h_{s}=h_{s}{ }^{\prime}+x_{s}\left(h_{s}{ }^{\prime}-h_{s}{ }^{\prime}\right)
$$

A compressor efficiency $\eta_{c}$ is given in terms of the discharge enthalpy $h_{d t h}$ calculated under the assumption of an isentropic process, the measured refrigerant mass flow-rate $G_{r}$, and the measured compressor power consumption $W_{c}$ :

$$
\eta_{c}=G_{r}\left(h_{d t h}-h_{s}\right) / W_{c}
$$

A discharge enthalpy $h_{d^{\prime}}$ calculated under the assumption of no heat radiation from the compressor is given in terms of compressor efficiency $\eta_{c}$ :

$$
h_{d^{\prime}}=h_{s}+W_{c} / G_{r}=h_{s}+\left(h_{d t h}-h_{s}\right) / \eta_{c}
$$

The discharge enthalpy $h_{d 2}$ estimated from the assumed value of suction quality $x_{s}$ is represented as follows, letting $Q_{a}$ be the practical heat radiation from the compressor:

$$
h_{d 2}=h_{d^{\prime}}-Q_{a} / G_{r}
$$

The heat radiation from the compressor $Q_{a}$ is modeled as follows, using $K A$ value ( $K$ : overall heat transfer coefficient, $A$ : heat transfer area) of heat radiation from the compressor $K A_{c}$ and the ambient temperature $T_{a}$ :

$$
Q_{a}=K A_{c}\left(T_{d}-T_{a}\right)
$$




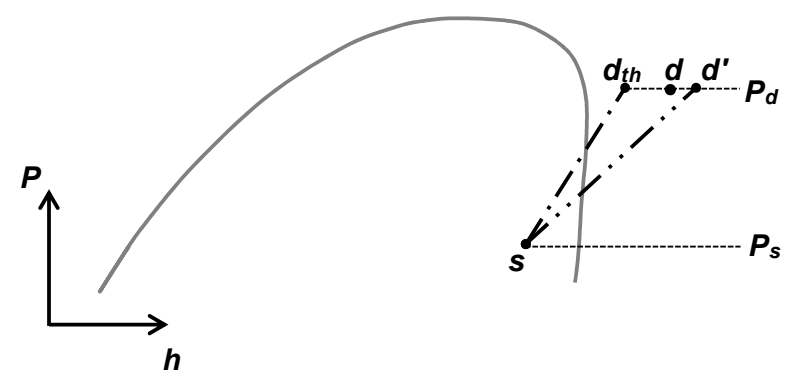

\begin{abstract}
s: Practical state point of compressor suction;
d: Practical state point of compressor discharge;

$\boldsymbol{d}_{t h}$ : State point of compressor discharge to which refrigerant changes from compressor suction state point $s$ under assumption of isentropic process; $\boldsymbol{d}^{\prime}$ : State point of compressor discharge to which refrigerant changes from compressor suction state point $s$ under assumption of no heat radiation from compressor.
\end{abstract}

Fig. 1 Relationships between enthalpies at compressor suction and discharge.

The suction quality $x_{s}$ can be identified and estimated in such a way that the enthalpy $h_{d 2}$ in Eq. (8) is the same as the enthalpy $h_{d}$ calculated from the discharge pressure $P_{d}$ and temperature $T_{d}$.

\subsection{Estimation of Suction Quality by Measuring Liquid and Vapor Flow-Rates}

This method is the conventional one used in the compressor test setup. In this study, the air conditioner test setup is constructed so that liquid and vapor flow-rates can be measured. The suction quality can be estimated by reproducing the refrigeration cycle in such a way that the suction pressure $P_{s}$, discharge pressure $P_{d}$, and discharge temperature $T_{d}$ are the same as those during the practical operation.

Fig. 2 shows the configurations of refrigeration cycle and $P$ - $h$ diagrams in the practical operation test and reproduction test. In the refrigeration cycle of the reproduction test, part of the liquid refrigerant from the condenser outlet is bypassed, then depressurized, and finally returned to the compressor suction line, joining the superheated vapor refrigerant through the evaporator. The bypassed liquid flow-rate is controlled with a pressure reducing valve in such a way that pressure $P_{s}$, pressure $P_{d}$, and temperature $T_{d}$ are the same as those of the refrigeration cycle of the practical operation test. Let $h_{s v}$ be the superheated vapor enthalpy, $h_{b l}$ the bypassed liquid enthalpy, $G_{r}$ the total refrigerant mass flow-rate, and $G_{b l}$ the bypassed refrigerant mass flow-rate. Then the suction enthalpy $h_{s}$ is given by:

$$
h_{s}=\frac{h_{s v}\left(G_{r}-G_{b l}\right)+h_{b l} G_{b l}}{G_{r}}
$$

The suction quality $x_{s}$ is obtained with Eq. (4).

\subsection{Expression for Estimating Compressor Suction State}

A relational expression for estimating a compressor suction state from a discharge state is discussed below. The state point $s_{t h}$ is newly defined as the state of compressor suction from which the refrigerant changes to the practical state point of the compressor discharge $d$ under the assumption of an isentropic process. Fig. 3 shows relationships between enthalpies at the compressor suction and discharge in this case. The suction enthalpy $h_{s}$ is represented as follows, using the discharge enthalpy $h_{d}$, the isentropic compression work based at the practical state point of the compressor discharge $\left(h_{d}-h_{s t h}\right)$, and the estimating coefficient for the suction state $\eta_{c}^{\prime}$.

$$
h_{s}=h_{d}-\left(h_{d}-h_{s t h}\right) / \eta_{c}{ }^{\prime}
$$

Eq. (11) can be transformed with respect to $\eta_{c}{ }^{\prime}$ as follows: 


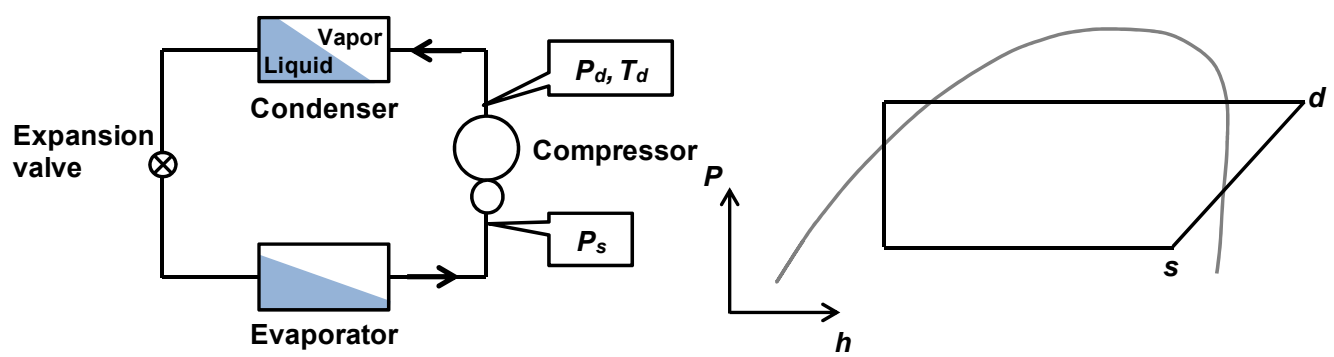

(1) Practical operation test

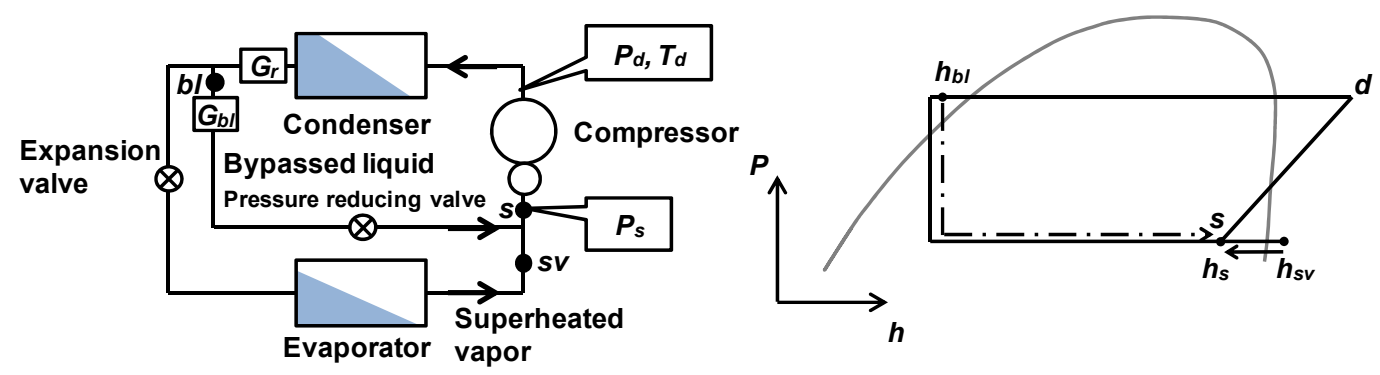

(2) Reproduction test

Fig. 2 Configurations of refrigeration cycle and $P$ - $h$ diagrams.

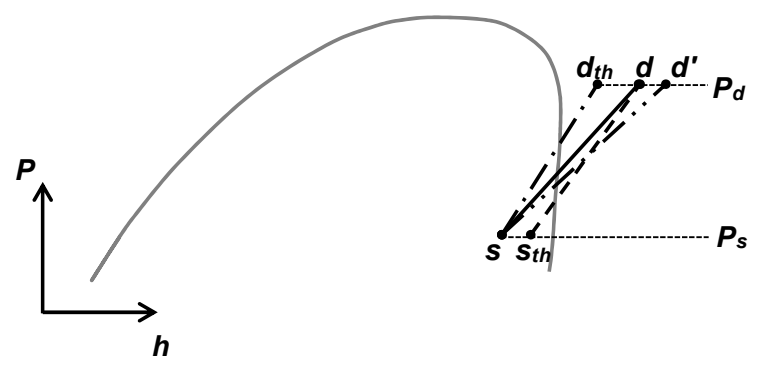

s: Practical state point of compressor suction;

$\boldsymbol{d}$ : Practical state point of compressor discharge;

$\boldsymbol{d}_{t h:}$ : State point of compressor discharge to which refrigerant changes from

compressor suction state point $s$ under assumption of isen tropic process;

$\boldsymbol{d}$ ': State point of compressor discharge to which refrigerant changes from

compressor suction sate point $s$ under assumption of no heat radiation from compressor;

$\boldsymbol{s}_{t h}$ : State point of compressor suction from which refrigerant changes to

compressor discharge state point $\boldsymbol{d}$ under assumption of isentropic process.

Fig. 3 Relationships between enthalpies at compressor suction and discharge with $s_{t h}$ added.

$$
\begin{gathered}
\eta_{c}{ }^{\prime}=\frac{h_{d}-h_{s t h}}{h_{d}-h_{s}} \\
=\frac{h_{d}-h_{s t h}}{h_{d t h}-h_{s}} \frac{h_{d^{\prime}}-h_{s}}{h_{d}-h_{s}} \frac{h_{d t h}-h_{s}}{h_{d^{\prime}}-h_{s}}
\end{gathered}
$$

where, $h_{d^{\prime}}$ is the discharge enthalpy under the assumption of no heat radiation from the compressor and $h_{d t h}$ is the discharge enthalpy under the assumption of the isentropic process from the suction enthalpy $h_{s}$, as described before. The first ratio in Eq. (12) is (the isentropic compression work with regard to the practical state point of the compressor discharge)/(the isentropic compression work with regard to the practical state point of the compressor suction), the second ratio is (the enthalpy difference 
between the suction and the discharge under the assumption of no heat radiation from the compressor)/(the enthalpy difference between the suction and the practical discharge), and the third ratio is the compressor efficiency $\eta_{c}$. Let $k_{w}$ be the first ratio, which is related to the isentropic compression work, $k_{r}$ be the second ratio, which is related to the radiation heat from the compressor, and $k_{p}$ be the product of these ratios. Then the estimating coefficient for the suction state $\eta_{c}{ }^{\prime}$ is represented as follows:

$$
\eta_{c}{ }^{\prime}=k_{w} k_{r} \eta_{c}=k_{p} \eta_{c}
$$

Let $h_{d}=h_{d 2}$ in Eq. (8), and then $k_{r}$ is transformed as follows using Eq. (8):

$$
k_{r}=\frac{h_{d^{\prime}}-h_{s}}{h_{d}-h_{s}}=\frac{\left(h_{d}-h_{s}\right)+Q_{a} / G_{r}}{h_{d}-h_{s}}
$$

Values of $k_{w}$ and $k_{r}$ are both larger than 1 .

To evaluate the validity of the method to estimate the suction quality by applying energy balances on the compressor, this method will be compared below with the conventional method by measuring the liquid and vapor flow-rates in a room air conditioner test. The characteristics of the coefficients in the relational expression for estimating the compressor suction state calculated from the test results will also be discussed.

\section{Experimental Setup and Test Procedure}

A room air conditioner used for the test is one for R410A with a rated cooling and heating capacities of $7.1 \mathrm{~kW}$ and $8.5 \mathrm{~kW}$, respectively. Fig. 4 shows the system diagram of the experimental setup. The compressor is an inverter-driven scroll compressor. R410A was replaced with $\mathrm{R} 32$, and refrigeration oil developed for R32 was used. The air conditioner was modified for the experimental setup as to bypass the line before an expansion valve and connect it with the compressor suction line through a pressure reducing valve. Pressures and temperatures of the refrigerant were measured with strain gauge pressure transducers and sheath type thermocouples, respectively. A total mass flow-rate and a bypassed flow-rate of the refrigerant are measured with Coriolis flowmeters. The power consumption of the compressor was measured with a power meter.

In the experiment, first the practical operation test was conducted by closing a bypass valve. The reproduction test was then conducted by opening the bypass valve and controlling the bypass flow-rate with the pressure reducing valve in such a way that the suction pressure $P_{s}$, discharge pressure $P_{d}$ and temperature $T_{d}$ were the same as those of the practical operation test. At this time, to obtain the enthalpy of the refrigerant through an evaporator and a 4-way valve, the expansion valve was controlled in such a way that the refrigerant was superheated at the superheated temperature of $8{ }^{\circ} \mathrm{C}$. In this case, the refrigerant state in the evaporator was different from that in the practical operation test, and this resulted in a difference between the suction pressure $P_{S}$ and that in the practical operation. The air temperature conditions were then adjusted to match the $P_{s}$.

Table 1 shows test conditions. Outdoor air temperatures for heating operation were $-15{ }^{\circ} \mathrm{C}$ and $-20{ }^{\circ} \mathrm{C}$, under which discharge temperatures of the compressor become very high. Discharge temperatures were varied by adjusting the expansion valve at each compressor rotational speed.

The suction qualities were calculated with measured values in the reproduction test with the methods by applying energy balances on the compressor and by measuring flow-rates. REFPROP (reference fluid properties) [8] was used for the thermodynamic properties of $\mathrm{R} 32$. The average value of heat radiation coefficients from the compressor $K A_{c}$ calculated from Eqs. (5)-(9) under the heating condition at the outdoor air temperature of $7{ }^{\circ} \mathrm{C}$, where the discharge temperature is not very high and the suction state can be set to be superheated, was used as $K A_{c}$ in the method by applying energy balances. A compressor ambient temperature was measured with a thermocouple installed near the compressor inside the outdoor unit. 
Fig. 4 System diagram of experimental setup.

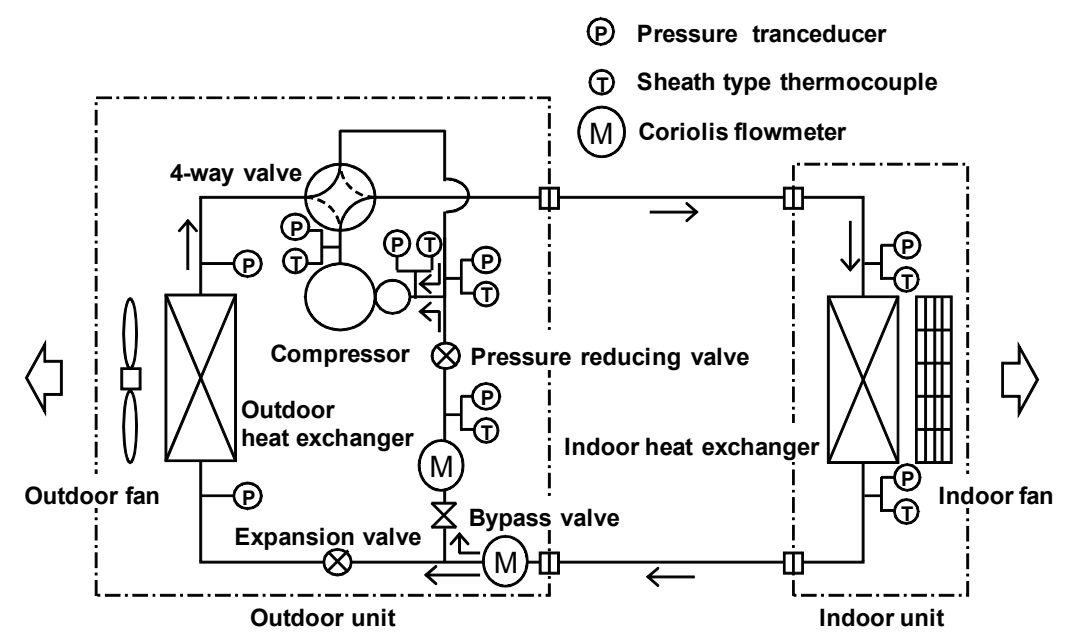

Table 1 Test conditions.

\begin{tabular}{lllll}
\hline \multicolumn{2}{c}{ Operation } & \multicolumn{3}{c}{ Heating } \\
\hline Indoor air & Dry bulb $\left({ }^{\circ} \mathrm{C}\right)$ & 20 & \\
temperature & Wet bulb $\left({ }^{\circ} \mathrm{C}\right)$ & 15 & \\
Outdoor air & Dry bulb $\left({ }^{\circ} \mathrm{C}\right)$ & $-15,-20$ & \\
temperature & Wet bulb $\left({ }^{\circ} \mathrm{C}\right)$ & - & \\
\hline $\begin{array}{l}\text { Compressor rotational speed } \\
\left(\text { min }^{-1}\right)\end{array}$ & 3,250 & 5,350 & 7,150 \\
Indoor fan rotational speed & Low & Medium & High \\
Outdoor fan rotational speed & Medium & Medium & High \\
\hline
\end{tabular}

\section{Experimental Results and Discussions}

\subsection{Comparisons of Estimated Vapor Qualities at Compressor Suction}

Fig. 5 compares suction qualities $x_{s}$ estimated with methods by applying energy balances on the compressor and by measuring flow-rates. Suction qualities estimated with the energy balancing method agree well with those estimated with the flow-rate measuring method within $\pm 0.5 \%$ in the quality range of 0.86-1.01 at each air temperature and each compressor rotational speed. The energy balancing method can estimate the suction quality as precisely as the flow-rate measuring method without adding the liquid bypass line to the original refrigeration cycle and is therefore effective.

\subsection{Characteristics of Coefficients in Relational} Expression for Estimating Compressor Suction State

This subsection will discuss the characteristics of the coefficients in the relational expression for estimating the compressor suction state calculated from the test results. Fig. 6 shows the relationships among suction quality, estimating coefficient, and its related ratios. The discharge temperature $T_{d}$ decreases as suction quality $x_{s}$ decreases. $T_{d}$ increases as compressor rotational speed increases, because the pressure ratio of the compressor also increases. The temperature barely rises between the compressor rotational speeds of 5,350 $\mathrm{min}^{-1}$ and 7,150 $\mathrm{min}^{-1}$, because the indoor and outdoor fan rotational speeds are increased. The compressor efficiency $\eta_{c}$ decreases as rotational speed increases and suction quality $x_{s}$ decreases. $\eta_{c}$ also decreases as outdoor air temperature decreases. One reason for $\eta_{c}$ decreasing as $x_{s}$ decreases is that the increase in the amount of liquid refrigerant in the compression chamber as $x_{s}$ decreases leads to the increase in the amount of the refrigerant that dissolves in the refrigeration oil and the decrease in the viscosity of the oil. This results in the decrease in the sealing performance in the compressor chamber.

The ratio $k_{w}\left(=\left(h_{d}-h_{s t h}\right) /\left(h_{d t h}-h_{s}\right)\right)$ increases as compressor rotational speed increases and suction quality $x_{s}$ decreases. This is because the decrease in the compressor efficiency $\eta_{c}$ as compressor rotational speed increases and $x_{s}$ decreases leads to the increase in the difference between $h_{d t h}$ and $h_{d} . k_{w}$ also increases 

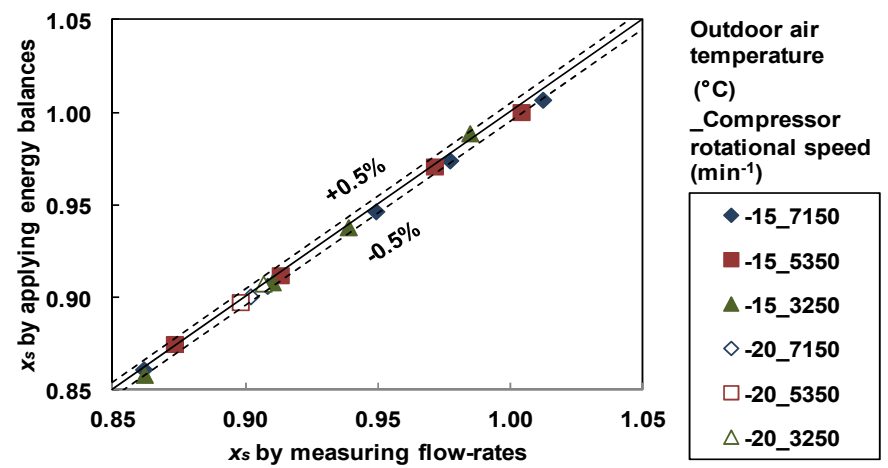

Fig. 5 Comparisons of suction qualities estimated with methods by applying energy balances and measuring flow-rates.
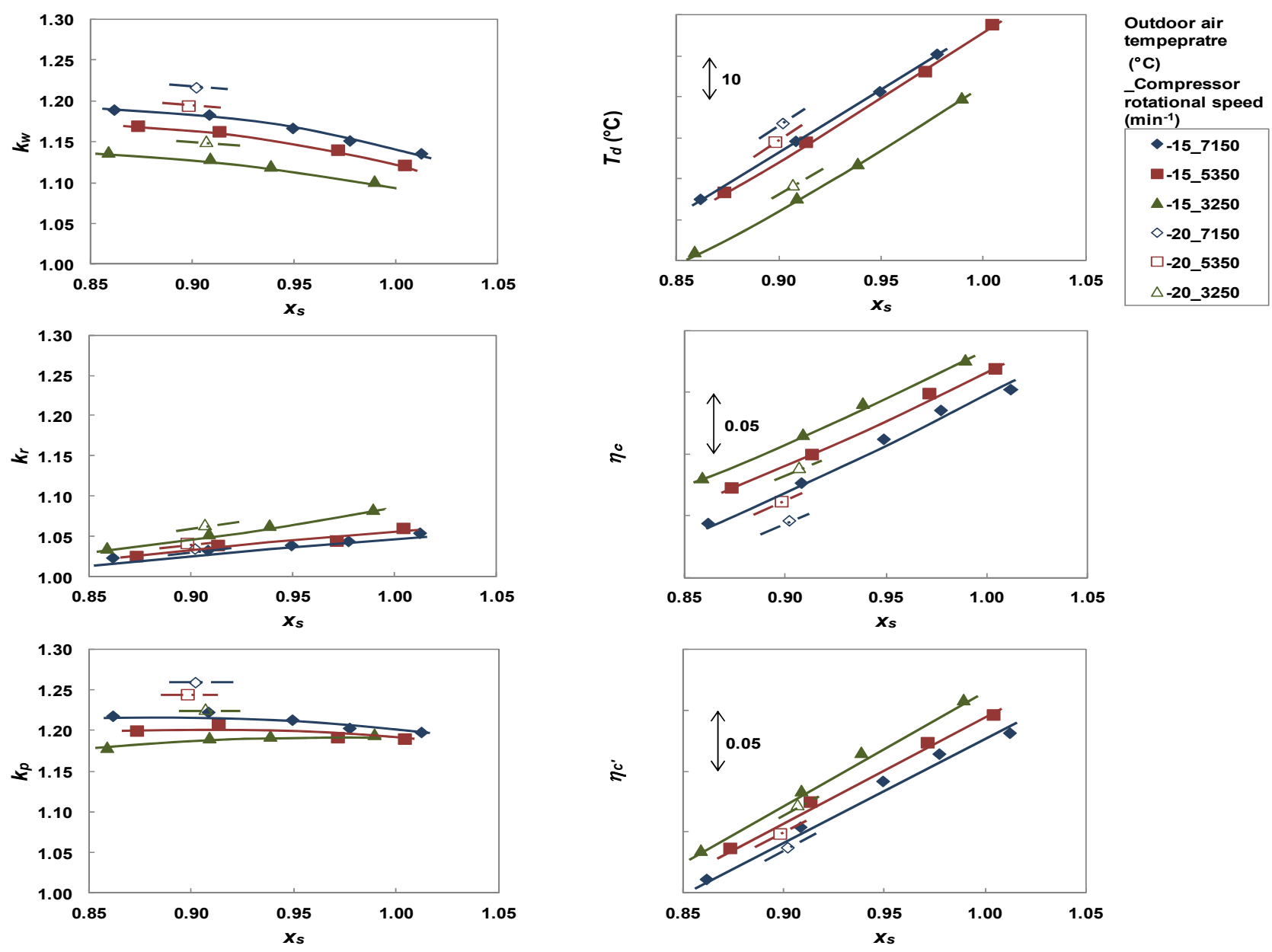

Fig. 6 Relationships between suction qualities and estimating coefficients

as outdoor air temperature decreases, which leads to the decrease in $\eta_{c}$.

The characteristics of the ratio $k_{r}$ (= $\left.\left(h_{d^{\prime}}-h_{s}\right) /\left(h_{d}-h_{s}\right)\right)$ will be explained with reference to Eqs. (9) and (14). The refrigerant flow-rate $G_{r}$ increases as compressor rotational speed increases and
$1 / G_{r}$ in Eq. (14) then decreases. Therefore, $k_{r}$ decreases. The decrease in $T_{d}$ as $x_{s}$ decreases leads to the decrease in the heat radiation from the compressor $Q_{a}$, resulting in the decrease in $k_{r}$. As outdoor air temperature decreases, the increase in the difference between $T_{d}$ and the ambient temperature $T_{a}$ leads to 
the increase in $Q_{a}$ and $G_{r}$ decreases. Therefore, $k_{r}$ increases.

The range of product $k_{p}$ becomes small, because the tendencies of $k_{w}$ and $k_{r}$ are opposite with regard to the compressor rotational speed and $x_{s}$. Especially with changing $x_{s}, k_{p}$ barely changes and is nearly constant. As compressor rotational speed increases, $k_{p}$ increases. $k_{p}$ also increases as outdoor air temperature decreases. $\eta_{c}{ }^{\prime}$ that is the product of $\eta_{c}$ and $k_{p}$ decreases as $x_{s}$ decreases. The range of $\eta_{c}{ }^{\prime}$ is smaller than that of $\eta_{c}$ with regard to the compressor rotational speed, because $\eta_{c}$ decreases and $k_{p}$ increases as compressor rotational speed increases.

\section{Conclusions}

From this study, it can be concluded that:

- Suction qualities estimated with the method by applying energy balances on the compressor agree well with those estimated with the conventional method by measuring liquid and vapor flow-rates;

- The energy balancing method can estimate the suction quality without adding the liquid bypass line to the original refrigeration cycle and is therefore effective;

- The suction enthalpy is represented by using the discharge enthalpy, the isentropic compression work based at the practical state point of the compressor discharge, the compressor efficiency, the ratio related with isentropic compression work, and the ratio related with the heat radiation from the compressor;

- The product of the ratios is nearly constant as suction quality changes.

\section{References}

[1] The European Parliament and the Council of the European Union. 2014. "Regulation (EU) No. 517/2014 of the European Parliament and of the Council of 16 April 2014 on Fluorinated Greenhouse Gases and Repealing Regulation (EC) No 842/2006." Official Journal of the European Union 57 (5): 195-230.

[2] The METI (Ministry of Economy, Trade and Industry), Japan. 2013. "Cabinet Decision on the Bill for the Act for Partial Revision of the Act on Ensuring the Implementation of Recovery and Destruction of Fluorocarbons concerning Designated Products.” News Release of METI. Accessed February 28, 2015. http://www.meti.go.jp/english/press/2013/0419_01.html.

[3] The JRAIA (Japan Refrigeration and Air Conditioning Industry Association). 2013. "Movement to R32 Refrigerant in the Japanese Market." JRAIA News. Accessed February 28, 2015. http://www.jraia.or.jp/ english//about/ letter/Vol.1304\%20NL.pdf.

[4] Yajima, R., Kita, K., Taira, S., and Domyo, N. 2000. "R32 as a Solution for Energy Conservation and Low Emission." In Proceedings of the International Refrigeration and Air Conditioning Conference, 407-14.

[5] Afjei, T., Suter, P., and Favrat, D. 1992. "Experimental Analysis of an Inver-Driven Scroll Compressor with Liquid Injection." In Proceedings of the International Compressor Engineering Conference, 541-50.

[6] Hirano, T., Hagimoto, K., and Matsuda, S. 1993. "Study on Scroll Compressor Behavior in Case of Liquid Refrigerant Injection.” Trans. JAR 10 (2): 227-38.

[7] Dutta, A. K., Yanagisawa, T., and Fukuta, M. 1996. “A Study on Compression Characteristic of Wet Vapor Refrigerant." In Proceedings of the International Compressor Engineering Conference, 235-40.

[8] Lemmon, E. W., Huber, M. L., and McLinden, M. O. 2013. NIST Standard Reference Database 23: Reference Fluid Thermodynamic and Transport Properties-REFPROP, Version 9.1. Gaithersburg: National Institute of Standards and Technology. 